\title{
Homoharringtonine acts synergistically with SG235- TRAIL, a conditionally replicating adenovirus, in human leukemia cell lines
}

\author{
Hai-tao MENG ${ }^{1}$, Lu LI ${ }^{1}$, Hui LIU ${ }^{1}$, Ying WANG ${ }^{1}$, Gong-chu LI², Wen-bin QIAN ${ }^{1,2, *}$ \\ ${ }^{1}$ Institute of Hematology, the First Affiliated Hospital, College of Medicine, Zhejiang University, Hangzhou 310003, China; ${ }^{2}$ Xinyuan Ins- \\ titute of Medicine and Biotechnology, College of Life Sciences, Zhejiang Sci-Tech University, Hangzhou 310018, China
}

\begin{abstract}
Aim: To investigate the synergistic effects of SG235-TRAIL, a novel oncolytic adenovirus expressing tumor necrosis factor-related apoptosis-inducing ligand (TRAIL) and homoharringtonine (HHT) in human leukemia cell lines.

Methods: The combined effect of SG235-TRAIL and HHT was assessed using a crystal violet assay and 3-(4,5-dimethylthiazol-2-yl)-2,5diphenyltetrazolium bromide (MTT) assay, followed by combination index analysis. Cell apoptosis was measured using flow cytometry combined with fluorescein-isothiocyanate-Annexin $\mathrm{V}$ staining. The activation of caspase pathway and the expression of Bcl-2 family proteins, TRAIL, and E1A were examined using Western blotting.

Results: HHT synergized the cytotoxicity of SG235-TRAIL against leukemia cell lines Kasumi-1, KG-1, HL-60, and U937, concomitantly with increased apoptosis and enhanced activity of caspase-3 and -9. The combination therapy resulted in significantly lower levels of $\mathrm{Bcl}-2, \mathrm{Mcl}-1$, and Bid compared to treatment of cells with either HHT or SG235-TRAIL alone, suggesting that HHT sensitizes leukemia cells to SG235-TRAIL virus through alteration of anti-apoptotic signaling elements. Importantly, HHT combined with SG235-TRAIL did not show significant cytotoxicity to normal human mononuclear cells and mesenchymal stem cells.

Conclusion: Combining oncolytic adenovirus SG235-TRAIL and HHT synergistically enhances cytotoxicity in leukemia cells in vitro, suggesting that the combination therapy could represent a rational approach for the treatment of leukemia.
\end{abstract}

Keywords: homoharringtonine; oncolytic adenovirus; TRAIL; synergy; apoptosis; Kasumi-1 cells; KG-1 cells; HL-60 cells

Acta Pharmacologica Sinica (2009) 30: 1529-1536; doi: 10.1038/aps.2009.147; published online 12 October 2009

\section{Introduction}

Conditionally replicating adenovirus (CRAd) is a promising approach for the treatment of neoplastic diseases refractory to conventional therapies ${ }^{[1,2]}$. Such viruses are modified to take advantage of tumor-specific alterations, allowing selective lytic replication within tumor cells. Genetic modifications made to CRAds include the use of tumor-specific promoters, such as AFP, TERT, and PSA, controlling the expression of genes involved in viral replication ${ }^{[3,4]}$, and deletion of viral genes encoding proteins which help to complete the viral lytic cycle in tumor cells, but not in normal cells ${ }^{[5]}$. It is shown that the deletion of E1B-55 kDa, which inhibits p53 and prevents apoptosis, allows selective adenoviral replication in various tumor cells and leukemia cells ${ }^{[6,7]}$. A 24-bp deletion of E1A gene prevents the binding of E1A to retinoblastoma $(\mathrm{Rb})$, resulting in release of E2F and subsequent viral replication ${ }^{[8,9]}$.

* To whom correspondence should be addressed.

E-mail qianwenb@yahoo.com.cn

Received 2009-05-24 Accepted 2009-09-03
Furthermore, modifications of viral fiber, such as Ad5/11 and Ad5/F35 chimeric fibers, help to transduce type 5 adenovirus utilizing the Coxsackie-adenovirus recptor (CAR) as the primary receptor for viral internalization into cancer cells with low CAR expression levels ${ }^{[10-12]}$. We have previously shown that a CRAd with E1B-55 kDa deletion and Ad5/F35 chimeric fiber, which express exogenous apoptosis inducing gene tumor necrosis factor-related apoptosis-inducing ligand (SG235-TRAIL), effectively kills leukemic cells and reduces the growth of leukemic xenografts ${ }^{[13,14]}$. These results suggest that SG235-TRAIL virus might be envisaged for the treatment of leukemia.

The efficacy of CRAds can be improved by combination with chemotherapeutic agents. ONYX-015, a first E1B-55 kDa gene-deleted adenovirus, in combination with cisplatin or 5-fluorouracil has already been tested in phase II clinical trial for the patients of head and neck carcinoma. In gastrointestinal carcinoma metastatic to the liver and unresectable pancreatic carcinoma, enhanced antitumor activity of ONYX015 has been observed when combined with leucovorin and 
5-fluorouracil ${ }^{[15-17]}$.

Homoharringtonine (HHT), a plant alkaloid, was first investigated in China and has been reported to be active in acute myelogenous leukemia (AML), chronic myelogenous leukemia (CML) and myelodysplastic syndrome (MDS) ${ }^{[18-21]}$. However, early phase I and II clinical studies in the United States document a high incidence of cardiovascular complications with higher-dose continuous-infusion schedules ${ }^{[2]}$. Thus, in order to reduce drug dosage, minish cytotoxic effects on normal cells, and enhance therapeutic efficacy, novel approaches are required to achieve the successful application of HHT in leukemia therapy. The present study uses four representative human AML cell lines Kasumi-1, KG-1, HL-60, and U937. The objective of this study was to investigate whether HHT could act synergistically with a new oncolytic virus SG235-TRAIL in treating leukemia cells.

\section{Materials and methods Cell lines}

Human AML cell lines Kasumi-1 and KG-1 were kindly provided by Prof SJ CHEN (Shanghai Jiaotong University, China) and Dr RZ XU (Zhejiang University, China), respectively. HL-60 and U937 cell lines were purchased from the American Type Culture Collection (Rockville, MD, USA). Cells were maintained in RPMI-1640 medium (Hyclone Laboratories, Logan, UT, USA) supplemented with $10 \%$ fetal bovine serum (FBS; Life Technologies, Inc, Grand Island, NY, USA) and 1\% L-glutamine (Life Technologies, Inc) at $37^{\circ} \mathrm{C}$ in $5 \% \mathrm{CO}_{2}$ humid atmosphere.

Human mesenchymal stem cells isolation and culture conditions Human mesenchymal stem cells (MSCs) and mononuclear cells (MNCs) were obtained from bone marrow samples from healthy donors after informed consent. Bone marrow mononuclear cells were isolated by Ficoll density gradient and cultured in DMEM medium (Gibco-BRL, Grand Island, NY, USA) containing $10 \%$ FBS. After $3 \mathrm{~d}$, nonadherent cells were discarded and adherent cells were replenished with fresh medium twice a week. When the culture reached confluency, MSCs were detached and identified by flow cytometry using the following mAbs: CD29-FITC, CD90-FITC, CD44-PE (all from eBioscience Inc, San Diego, CA, USA), and CD166-PE (from R\&D Systems, Minneapolis, MN, USA). MSCs were used for experiments between the $3 \mathrm{rd}$ and 4 th passages.

\section{Construction of recombinant adenoviral vectors}

The construction of SG235-TRAIL has been described previously ${ }^{[13]}$. Plasmid pZD55-TRAIL was cotransfected with a serotype 35 adenovirus vector (pPE35) into 293 cells to generate the SG235-TRAIL by homologous recombination.

\section{Cell cytotoxicity assay}

Cytotoxicity on leukemia cells was determined by lactate dehydrogenase (LDH) activity released from the cytosol of damaged cells using a LDH detection kit (Roche, Philadelphia, PA, USA). Briefly, leukemia cells were seeded into 96-well plates at $2.0 \times 10^{5}$ cells/well. After treating cells with HHT, SG235-TRAIL, or HHT combined with SG235-TRAIL at different dosages as indicated, the cytotoxicity at each dose was then measured in triplicate wells by the cytotoxicity detection kit following the manufacturer's instruction.

\section{MTT assay}

Cells were plated on 96 -well plates at $1.0 \times 10^{4}$ per well, then treated with viruses and/or HHT at the indicated dosages for different times. PBS was used as a control. The cell viability was determined by MTT assay as previously described ${ }^{[13]}$.

\section{Crystal violet assay}

MSC were grown to subconfluent and infected with SG235TRAIL at various multiplicities of infection (MOI) combined with HHT. Three days after treatment, cells were exposed to $2 \%$ crystal violet (Sigma, St Louis, MO, USA) in 20\% methanol for $15 \mathrm{~min}$, then washed with distilled water $\left(\mathrm{dH}_{2} \mathrm{O}\right)$, and documented as photographs. The presence of staining indicates the presence of living cells.

\section{Western blotting}

Control and treated cells were lysed at the indicated times with a lysis buffer containing $1 \mathrm{~mol} / \mathrm{L}$ Tris- $\mathrm{HCl}, \mathrm{pH} 6.8,2$ $\mathrm{mol} / \mathrm{L} \mathrm{NaCl}, 0.1 \mathrm{~mol} / \mathrm{L}$ EDTA, $0.05 \mathrm{~mol} / \mathrm{L}$ EGTA, 0.5\% NP-40 and $10 \mathrm{mmol} / \mathrm{L}$ PMSF. Protein concentration was determined using the Bradford method. Samples containing $50 \mu \mathrm{g}$ of total cellular protein were subjected to standard Western blotting analysis after SDS-PAGE. Positive antibody reactions were visualized with a horseradish peroxidase-conjugated secondary antibody (Santa Cruz, Santa Cruz, CA, USA) and an enhanced chemiluminescence detection system according to the protocol of the manufacturer. Primary antibodies included antibodies against caspase- $3,-8$, and -9 , poly (adenosine diphosphate-ribose) polymerase (PARP), Bcl-2, Bak, Bcl- $x \mathrm{~L}$, and Bid (from Cell Signaling Technology, Beverly, MA, USA). Anti-Mcl-1 antibody was provided by Biovision (Mountain View, CA, USA). Antibodies against E1A and TRAIL were purchased from BD PharMingen (SanDiego, CA, USA). Antiactin antibodies were purchased from Santa Cruz.

\section{Flow cytometry analysis}

Cells were treated with HHT, SG235-TRAIL alone, or HHT combined with SG235-TRAIL at the indicated concentrations, and then washed with cold PBS for twice followed by staining with Annexin V-FITC (BD Pharmingen). Cell suspension was analyzed on a FACSCalibur flow cytometer (Becton Dickinson, San Jose, CA, USA).

To determine transduction efficiency of the virus, Kasumi-1 and HL-60 cells were infected with SG235-GFP at an MOI of 20 for $72 \mathrm{~h}$, and then subjected to flow cytometric analysis.

\section{Statistics}

The combination index (CIN) analysis were based on the methods of Chou and colleagues ${ }^{[23,24]}$. HHT and SG235-TRAIL virus-induced cytotoxic synergy was analyzed with the use of 
CalcuSyn software (Biosoft, Cambridge, UK), and expressed as $\log _{10}(\mathrm{CIN})$ versus fraction affected. By this method, CIN $<0$ indicates synergy; $\mathrm{CIN}=0$ indicates an additive effect; and $\mathrm{CIN}>0$ indicates antagonism.

\section{Results}

Infection of SG235-TRAIL and expression of TRAIL in leukemia cells

We inserted TRAIL gene expressing cassette into SG235, a new replicative adenovirus that has both the E1B-55-kDa deletion and Ad5/F35 chimeric fiber (Figure 1A). Transduction efficiency of the adenovirus was evaluated by flow cytometric analysis. GFP-positive cells were $54.08 \%$ and $37.01 \%$, respectively, in Kasumi-1 and HL-60 cells treated with SG235-GFP at an MOI of 20 for $72 \mathrm{~h}$ (Figure 1B). To verify the infectivity of SG235-TRAIL in leukemia cells and the expression of TRAIL, Kasumi-1 cells were treated with $8 \mathrm{ng} / \mathrm{mL}$ of HHT, $20 \mathrm{MOI}$ of
SG235-TRAIL, or HHT combined with SG235-TRAIL. Cells received no treatment served as a negative control. As determined by Western blotting (Figure 1C), TRAIL and adenoviral E1A protein were detected in cells treated with SG235-TRAIL or HHT combined with SG235-TRAIL, but not in control or cells treated with HHT alone. Results indicated that SG235TRAIL successfully infected Kasumi-1 cells and expressed TRAIL in these cells. Furthermore, the expression levels of TRAIL and E1A were not significantly different between the treatments of cells with SG235-TRAIL alone and HHT combined with SG235-TRAIL, suggesting that the infection of SG235-TRAIL and the expression of TRAIL in leukemia cells were not altered by HHT treatment.

\section{Cytotoxicity of HHT combined with SG235-TRAIL on leukemia} cells

HHT (2-8 ng/mL) was combined with SG235-TRAIL (5-20

A
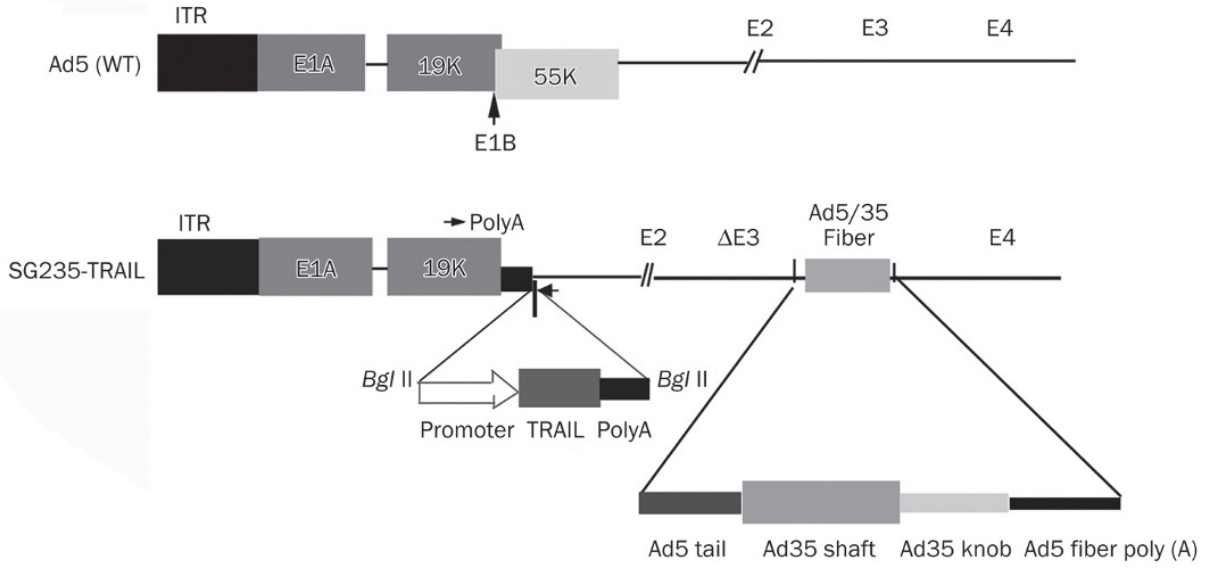

B
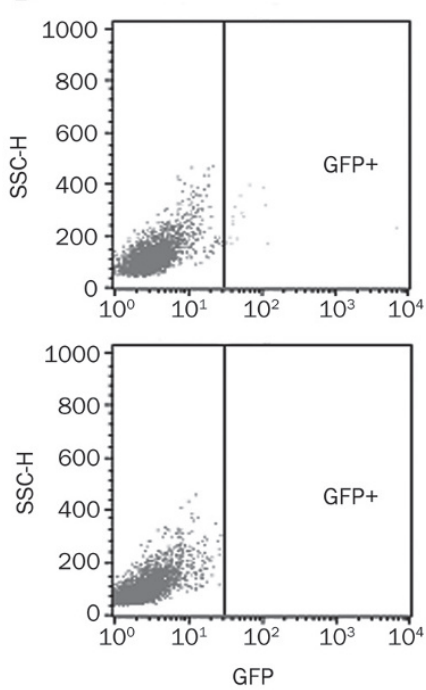

After
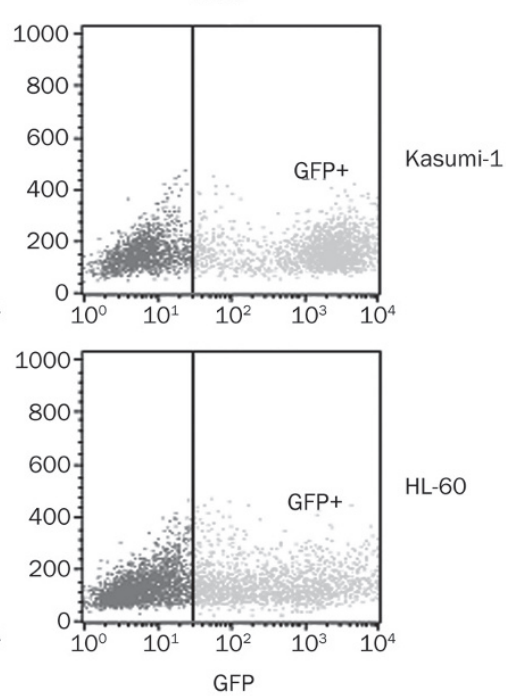

C

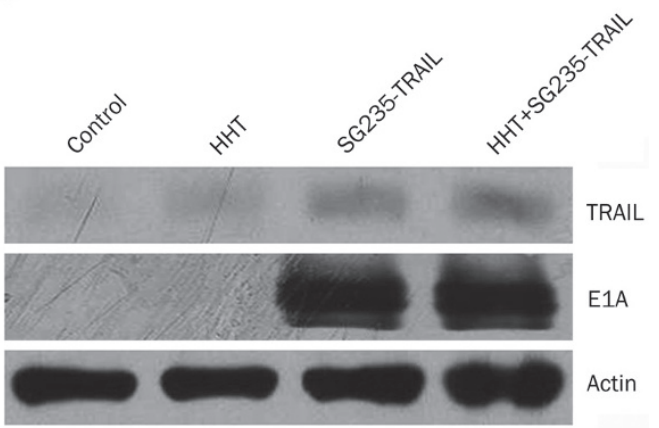

Figure 1. Schematic representation of SG235-TRAIL and infectivity of the viruses in leukemia cells. (A) Compared to wild-type Ad5, SG235-TRAIL has an E1B 55-kDa deletion and an Ad5/F35 chimeric fiber. The TRAIL gene was cloned in E2 region. (B) Kasumi-1 and HL-60 cells were infected with SG235-GFP at an MOI of 20 for $72 \mathrm{~h}$, followed by flow cytometric analysis. (C) Kasumi- 1 cells were seeded in 24 -well plates (2.0 $\times 10^{6}$ cells/well) and treated with $8 \mathrm{ng}$ of HHT, $20 \mathrm{MOI}$ of SG235-TRAIL, or $8 \mathrm{ng}$ of HHT combined with $20 \mathrm{MOI}$ of SG235-TRAIL. Cells were then harvested after $48 \mathrm{~h}$ and cell extractions were subjected to Western blot with antibodies against TRAIL, E1A, and Actin. One representative experiment of three performed is shown. 
MOI) to treat leukemia cell lines Kasumi-1, KG-1, HL-60, and U937, respectively. As shown in Figure 2A, treatment of cells with HHT combined with SG235-TRAIL resulted in a significantly lower cell viability than either HHT or SG235-TRAIL treatment, especially in cells treated with $8 \mathrm{ng} / \mathrm{mL}$ of HHT and/or $20 \mathrm{MOI}$ of SG235-TRAIL, which were determined by LDH assays. Similar results were obtained using MTT assay (data not shown). As shown in Figure 2B, HHT plus SG235TRAIL revealed synergic cytotoxicity $(\mathrm{CIN}<0)$ in three human AML cell lines (KG-1, HL-60, and U937). Whereas, the combination resulted in synergic cytotoxicity but only at relatively high concentrations (6 ng and $8 \mathrm{ng}$ for HHT, and $15 \mathrm{MOI}$ and 20 MOI for SG235-TRAIL) in Kasumi-1 cell line.

The cytotoxicity of HHT combined with SG235-TRAIL was further evaluated through Annexin V staining followed by flow cytometry analysis. As shown in Figure $3 \mathrm{~A}, 8 \mathrm{ng} / \mathrm{mL}$ of HHT combined with $20 \mathrm{MOI}$ of SG235-TRAIL resulted in $78.08 \%, 81.90 \%$, and $93.39 \%$ of Annexin V-positive cells in
Kasumi-1, KG-1, and U937 cells, respectively, whereas HHT alone caused $12.68 \%, 10.28 \%$, and $6.48 \%$ of Annexin V-positive cells, and SG235-TRAIL alone caused 39.99\%, 30.32\%, and $68.67 \%$ of Annexin V-positive cells, in Kasumi-1, KG-1, and U937, respectively. The data indicated that HHT significantly enhanced the cytotoxicity of SG235-TRAIL in leukemia cells.

HHT synergized the cytotoxicity of SG235-TRAIL in leukemia cells through affecting apoptotic signaling elements

To further investigate the underlying mechanism of the synergistic effect of HHT combined with SG235-TRAIL, apoptotic signaling elements were analyzed by Western blotting. As shown in Figure 3B, treating Kasumi-1 cells with 8 $\mathrm{ng} / \mathrm{mL}$ of HHT combined 20 MOI of SG235-TRAIL significantly increased levels of cleaved forms of caspase-9, -3, and PARP, as compared to treating cells with either HHT or SG235-TRAIL alone. The level of cleavage of caspase-8 was not significantly increased by HHT combined with SG235-

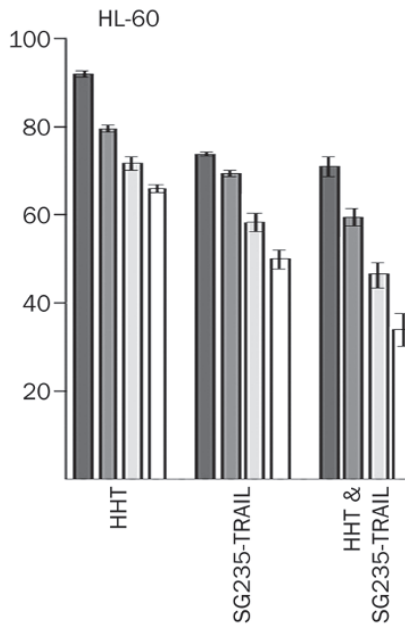

HL-60

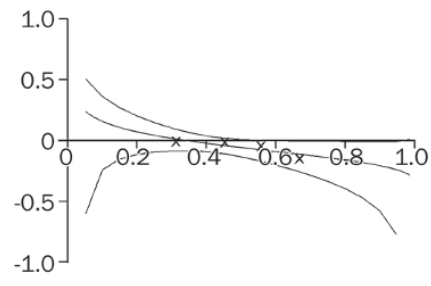

Fraction affected

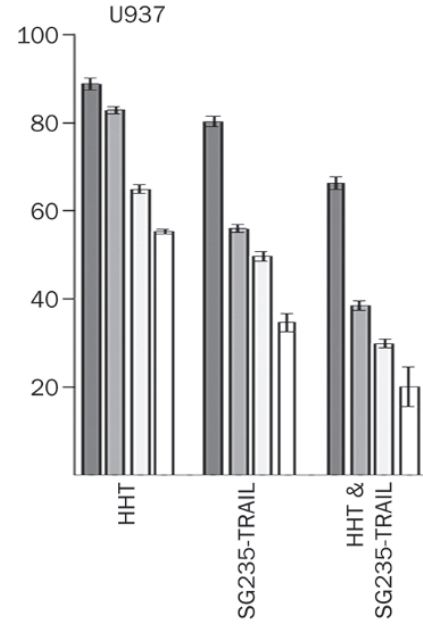

U937

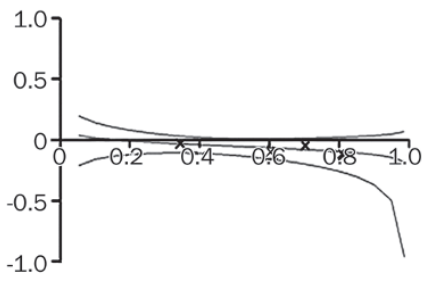

Fraction affected

Figure 2. Synergetic cytotoxicity of HHT combined with SG235-TRAIL. (A) Dose response of leukemic cells to HHT, SG235-TRAIL, and HHT combined with SG235-TRAIL. Kasumi-1, KG-1, HL-60, or U937 cells were seeded into 96-well plates and treated with HHT, SG235-TRAIL alone, or HHT combined with SG235-TRAIL at the dosages indicated. After $72 \mathrm{~h}$, cell viability was then measured by a cytotoxocity detection kit (LDH). The means and standard errors of results from three independent experiments are shown. (B) Synergistic effect of HHT combined with SG235-TRAIL. Synergy was quantified by combination index $(\mathrm{CIN})$ analysis and expressed as $\log _{10}(\mathrm{CIN})$ versus fraction affected. Where calculable, 95\% confidence intervals are shown. 
A
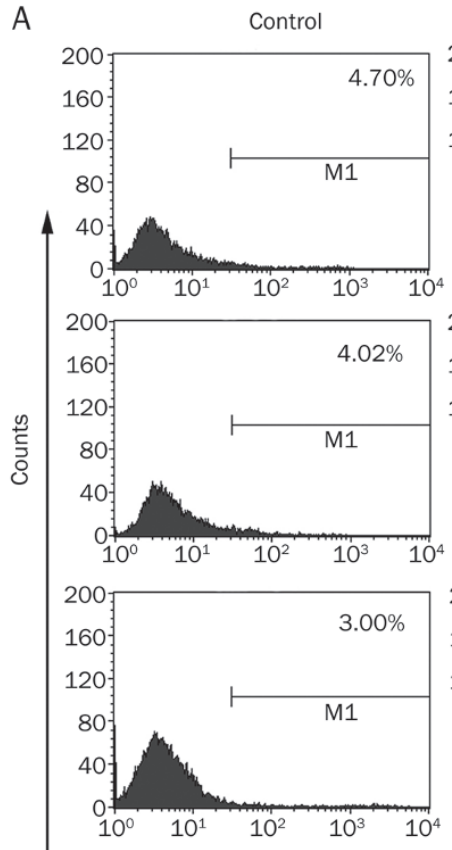

HHT
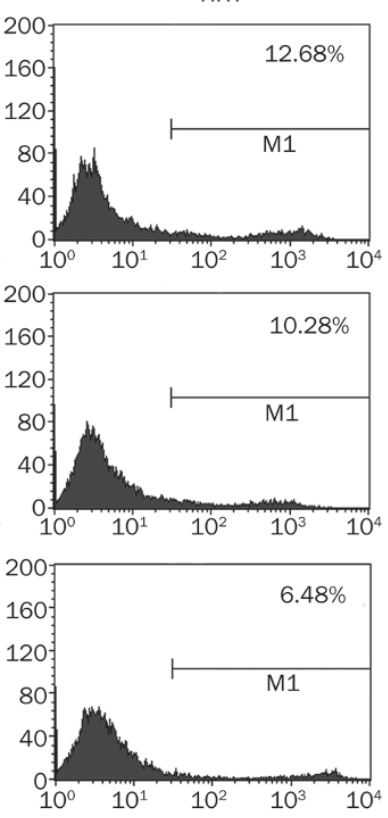

SG235
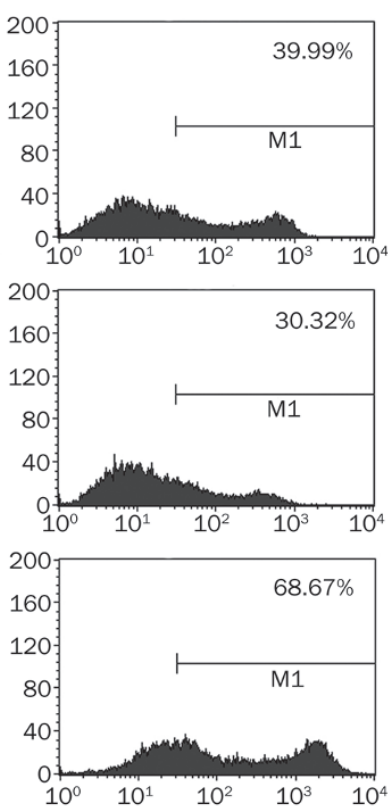

HHT+SG235-TRAIL

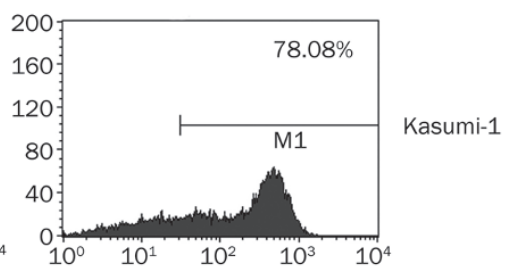

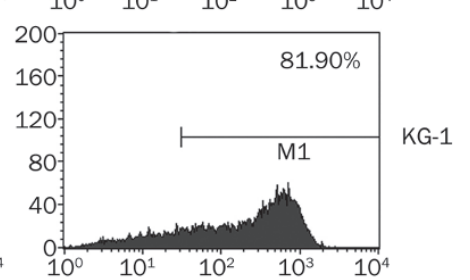

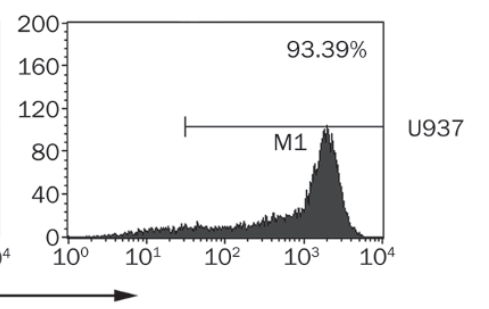

Annexin V-FITC

B

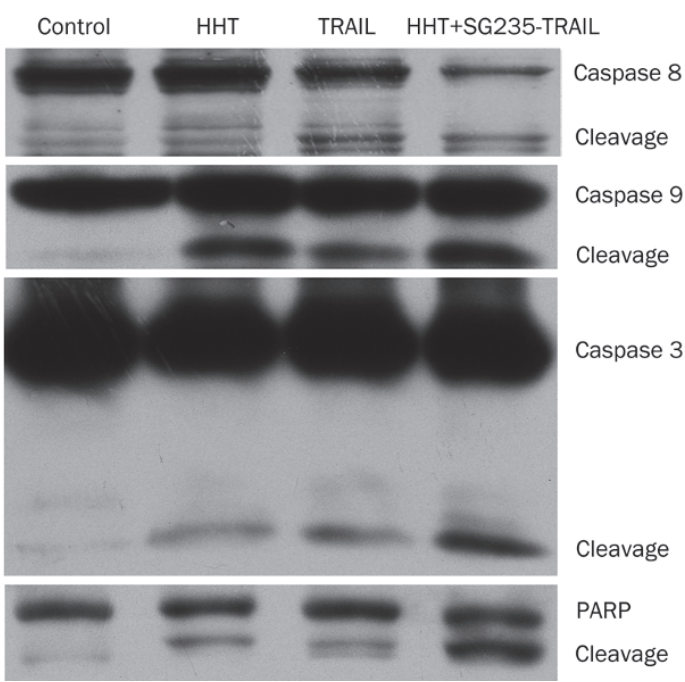

TRAIL. Our results suggest that HHT synergized the cytotoxicity of SG235-TRAIL through affecting the caspase pathway.

Bcl-2 family members involved in apoptosis were further investigated by Western blotting. As compared to negative control, $8 \mathrm{ng}$ of HHT treatment apparently down-regulated the Bcl- $\mathrm{x}_{\mathrm{L}}$ level in Kasumi-1 cells. However, the levels of Mcl-1, Bcl-2, Bak, and Bid were not affected (Figure 4). Treating cells with $20 \mathrm{MOI}$ of SG235-TRAIL significantly resulted in lower levels of Mcl-1 and Bcl- $\mathrm{x}_{\mathrm{L}}$, as well as an up-regulated level of Bak. The levels of Bid and Bcl-2 were not affected by the SG235-TRAIL treatment. Interestingly, treating cells with $8 \mathrm{ng}$ of HHT combined with 20 MOI of SG235-TRAIL resulted in apparently lower levels of Mcl-1, Bid, and Bcl-2, as compared to treating cells with either HHT or SG235-TRAIL.
Figure 3. HHT enhanced cell death and caspase activity induced by SG235-TRAIL. (A) Kasumi-1 cells were treated with $8 \mathrm{ng}$ of $\mathrm{HHT}, 20 \mathrm{MOI}$ of SG235-TRAIL, or HHT combined with SG235-TRAIL for $72 \mathrm{~h}$. Cells received PBS served as a negative control. The cells were harvested, washed with PBS, and stained with Annexin $\mathrm{V}$ followed by flow cytometry analysis. (B) After $48 \mathrm{~h}$, control and the treated cells were harvested, and then cell extraction was subjected to Western blot with antibodies against caspase-8, $-9,-3$, and PARP. Representative of three separate experiments.

These data suggest that the combination of HHT and SG235TRAIL induced enhanced apoptosis in leukemia cells through affecting anti-apoptotic signaling elements such as Mcl-1, Bid, and Bcl-2.

\section{Effect of HHT combined with SG235-TRAIL on normal human cells}

Next, we examined the effects of HHT combined with SG235TRAIL on normal human MNCs and bone marrow MSCs. Results showed that treating cells with $8 \mathrm{ng}$ of HHT or $20 \mathrm{MOI}$ of SG235-TRAIL resulted in 3\% and 5\% of cell growth inhibition after $48 \mathrm{~h}, 11 \%$ and $14 \%$ growth inhibition after $72 \mathrm{~h}$, respectively. Combination of HHT and SG235-TAIL resulted in $7 \%$ of cell growth inhibition after $48 \mathrm{~h}$ and $21 \%$ after $72 \mathrm{~h}$ 


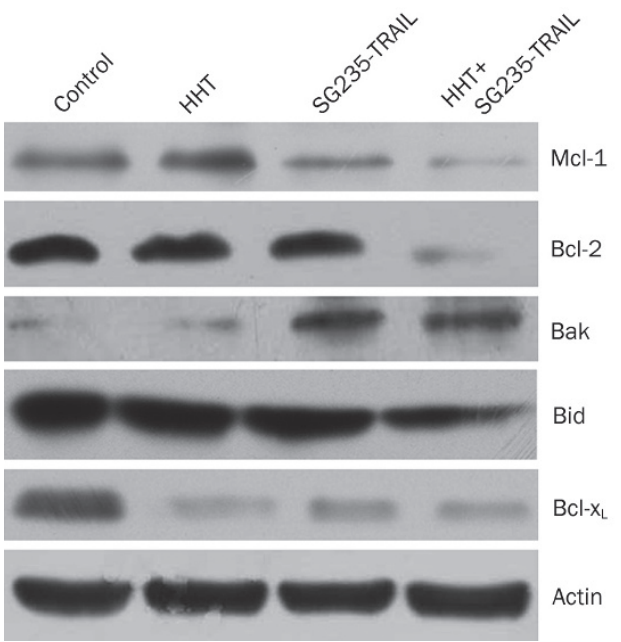

Figure 4. Effect of HHT combined with SG235-TRAIL on proapoptotic signaling elements in Kasumi-1 cells. Kasumi-1 cells were treated with or without $8 \mathrm{ng}$ of HHT, $20 \mathrm{MOI}$ of SG235-TRAIL, or $8 \mathrm{ng}$ of HHT combined with $20 \mathrm{MOI}$ of SG235-TRAIL. After $48 \mathrm{~h}$, cells were harvested and whole cell extracts were subjected to Western blot with antibodies against $\mathrm{Mcl}-1$, $\mathrm{Bcl}-2$, Bak, Bid, Bcl- $\mathrm{x}_{\mathrm{L}}$, and Actin. The data are representative of three determinations with identical results.

(Figure 5A). The crystal violet staining showed that combination of HHT and SG235-TRAIL at the indicated dosage did not apparently affect the viability of MSCs (Figure 5B). Our results suggest that HHT combined with SG235-TRAIL had limited cytotoxicity to normal human MNCs and almost no cytotoxicity to normal human MSC.

\section{Discussion}

CRAd represents a novel strategy for cancer therapies. However, oncolytic virotherapy has not been effective in complete tumor eradication in both preclinical animal models and clinical trials ${ }^{[25]}$. Several clinical studies have shown that an enhanced and even synergistic antitumor activity can be achieved when oncolytic adenoviruses are used in combination with chemotherapy ${ }^{[15-17]}$ or radiation therapy ${ }^{[26]}$, and suggested that the design of CRAd given in combination with cytotoxicitic chemotherapies will maximize the potential of oncolytic adenovirus and optimize patient benefit. We previously developed SG235, a new oncolytic adenovirus harboring an Ad5/F35 chimeric fiber and E1B-55-kDa deletion, and SG235-TRAIL, in which SG235 was armed with an antitumor gene, TRAIL ${ }^{[13]}$. We also demonstrated that both SG235 and SG235-TRAIL had significant antileukemia effects in vitro and in vivo ${ }^{[13,14]}$. Interestingly, SG235-TRAIL elicited a superior antileukemia activity as compared with SG235 ${ }^{[13]}$. In addition to the typical oncolytic toxicities, SG235-TRAIL induced apoptosis in leukemia cells through activating caspase-3 and $-9^{[13]}$. In this work, we found that HHT synergized with SG235TRAIL to inhibit proliferation of leukemia cells and induce apopotosis, suggesting that HHT could be used in combination with SG235-TRAIL in the treatment of leukemia patients.
A

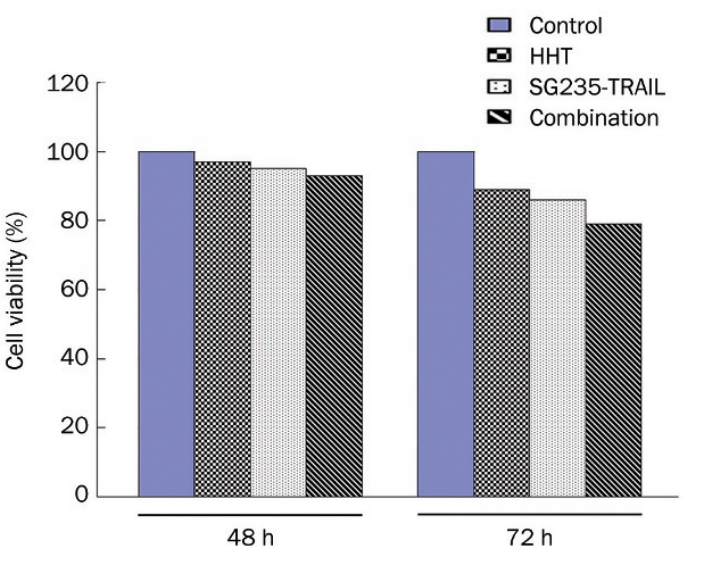

B

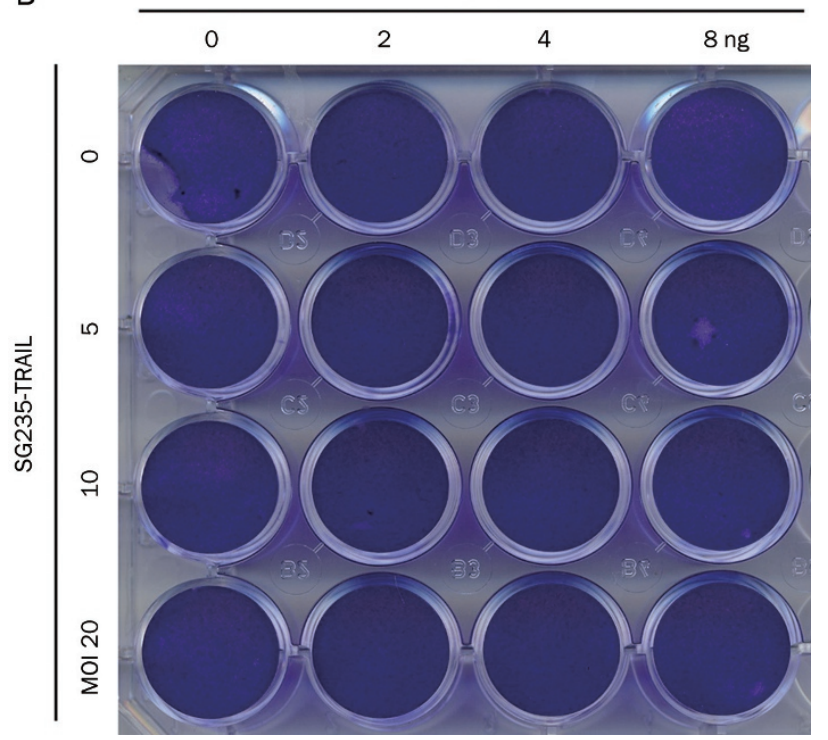

Figure 5. Effect of HHT combined with SG235-TRAIL on normal human mononuclear cells and mesenchymal stem cells. (A) Normal human mononuclear cells were treated with $8 \mathrm{ng}$ of HHT, $20 \mathrm{MOI}$ of SG235-TRAIL, or SG235-TRAIL plus HHT combination. Cells received PBS served as a negative control. After $48 \mathrm{~h}$ or $72 \mathrm{~h}$, cell viability was then measured by a MTT assay. Data are representative of three separate experiments. (B) Human mesenchymal stem cells were cultured on 24-well plates and treated with HHT and/or SG235-TRAIL at the indicated concentrations. After $72 \mathrm{~h}$, cell viability was measured by crystal violet assay. The images are representative of 3 independent experiments.

One of the rationales for the combination therapy is that the therapies have different safety profiles and therefore result in enhanced efficacy without increased adverse effects. Second, there is no overlapping resistance between oncolytic viruses and the other therapies. And finally, it may be possible to lower treatment doses, which will decrease possible unwanted toxicity ${ }^{[27]}$. The potential toxicity of SG235-TRAIL toward normal human cells is unknown, although previous clinic trails have indicated that oncolytic adenoviruses are safe ${ }^{[28]}$. In this study, we observed that $14 \%$ normal human MNCs were killed with the treatment of SG235-TRAIL at 20 MOI for $72 \mathrm{~h}$. 
However, normal MSCs were not affected by SG235-TRAIL, which is consistent with our previous results showing similar phenomena in normal human hematopoietic progenitor cells $^{[13]}$. HHT is a plant alkaloid with antileukemic activity. At high doses and with short infusion schedules, HHT has been associated with serious cardiovascular complications ${ }^{[22]}$. However, a lower-dose schedule of HHT $\left(2.5 \mathrm{mg} / \mathrm{m}^{2}\right.$ daily) has abrogated the occurrence of cardiovascular complications, which occurs in less than $5 \%$ of patients ${ }^{[29]}$. In this regard, we recently have demonstrated that HHT (4 mg daily) combined with cytarabine and aclarubicin result in high complete remission rate in patients with de novo AML. Only $2 \%$ patients have cardiovascular complications including tachycardia and palpitation $^{[30]}$. Together, the data indicate that reducing dose of HHT utilized will significantly minish toxicity to normal cells and tissues. It has been reported that LD50 of HHT in mice is $6.7 \mathrm{mg} / \mathrm{kg}$, and $\mathrm{IC}_{50}$ of HHT on HL-60 cells is 20 $\mathrm{ng} / \mathrm{mL}^{[31]}$. In this study, we found that HHT at lower concentration (up to $8 \mathrm{ng}$ ) could significantly synergize with SG235TRAIL for killing leukemia cells. The inhibition to MNCs by the combination was $21 \%$, but there was no toxicity toward normal MSCs. Together, these data suggest that SG235-TRAIL and HHT combination therapy may be safe, although this awaits confirmation in in vivo model systems.

The mechanism of this combination therapy remains elusive. HHT induces apoptosis in myeloid leukemia cells through affecting caspase-3 and Bax, a pro-apoptotic factor ${ }^{[18-21]}$. Overexpression of two antiapoptotic proteins $\mathrm{Bcl}-2$ and $\mathrm{Bcl}-\mathrm{x}_{\mathrm{L}}$ is commonly observed in many cancer types including leukemia, which is associated with chemotherapy resistance ${ }^{[32-34]}$. In addition, Bcl-2 family proteins are critical for the determination of sensitivity to TRAIL ${ }^{[35,36]}$. In mouse xenograft models, TRAIL exhibits potent antitumor activity and often synergizes with chemotherapy ${ }^{[37,38]}$. In this study, we observed that HHT induced down-regulation of $\mathrm{Bcl}-\mathrm{x}_{\mathrm{L}}$ protein. Interestingly, the combination of HHT with SG235-TRAIL resulted in significant lower levels of Bcl-2, Mcl-1, and Bid, which were not apparently affected by treating cells with either $8 \mathrm{ng}$ of HHT or 20 MOI of SG235-TRAIL alone. This might explain why HHT could synergize with SG235-TRAIL in the induction of leukemia cell death.

In summary, we have demonstrated for the first time that HHT could synergize with an oncolytic adenovirus SG235TRAIL to kill leukemia cells, without significant damage to normal human mononuclear cells and bone marrow mesenchymal stem cells. These data suggest that the combination therapy with HHT and oncolytic adenovirus could represent a rational approach for the treatment of leukemia.

\section{Acknowledgements}

This work was supported by National Natural Science Foundation of China grants 30470745 (Wen-bing QIAN); the Key Social Development Project of Zhejiang Province grants 2004c23005 (Wen-bing QIAN).

\section{Author contribution}

Wen-bin QIAN designed research; Hai-tao MENG, Lu LI, Ying WANG, Hui LIU, Gong-chu LI performed research; Hui LIU analyzed data; Wen-bin QIAN and Gong-chu LI wrote the paper.

\section{References}

1 Kirn D, Martuza RL, Zwiebel J. Replication-selective virotherapy for cancer: biological principles, risk management and future directions. Nat Med 2001; 7: 781-7.

2 Hawkins LK, Lemoine NR, Kirn D. Oncolytic biotherapy: a novel therapeutic platform. Lancet Oncol 2002; 3: 17-26.

3 Robson T, Hirst DG. Transcriptional targeting in cancer gene therapy. J Biomed Biotechnol 2003; 2003: 110-37.

4 Sadeghi H, Hitt MM. Transcriptionally targeted adenovirus vectors. Curr Gene Ther 2005; 5: 411-27.

5 Liu TC, Kim D. Viruses with deletions in antiapoptotic genes as potential oncolytic agents. Oncogene 2005; 24: 6069-79.

6 Bischoff JR, Kim DH, Williams A, Heise C, Horn S, Muna M, et al. An adenovirus mutant that replicates selectively in p53-deficient human tumor cells. Science 1996; 274: 373-6.

7 Qian W, Liu J, Tong Y, Yan S, Yang C, Yang M, et al. Enhanced antitumor activity by a selective conditionally replicating adenovirus combining with MDA-7/interleukin-24 for B-lymphoblastic leukemia via induction of apoptosis. Leukemia 2008; 22: 361-9.

8 Fueyo J, Gomez-Manzano C, Alemany R, Lee PS, McDonnell TJ, Mitlianga $\mathrm{P}$, et al. A mutant oncolytic adenovirus targeting the $\mathrm{Rb}$ pathway produces anti-glioma effect in vivo. Oncogene 2000; 19: 2-12.

9 Heise C, Hermiston T, Johnson L, Brooks G, Sampson-Johannes A, Williams A, et al. An adenovirus E1A mutant that demonstrates potent and selective systemic anti-tumoral efficacy. Nat Med 2000; 6: 1134-9.

10 Gaggar A, Shayakhmetov DM, Lieber A. CD46 is a cellular receptor for group B adenoviruses. Nat Med 2003; 9: 1408-12.

11 Tuve S, Wang H, Ware C, Liu Y, Gaqqar A, Bernt K, et al. A new group $B$ adenovirus receptor is expressed at high levels on human stem and tumor cells. J Virol 2006; 80: 12109-20.

12 Reddy PS, Ganesh S, Yu DC. Enhanced gene transfer and oncolysis of head and neck cancer and melanoma cells by fiber chimeric oncolytic adenoviruses. Clin Cancer Res 2006; 12: 2869-78.

13 Jin J, Liu H, Yang C, Li G, Liu X, Qian Q, et al. Effective gene-viral therapy of leukemia by a new fiber chimeric oncolytic adenovirus expressing TRAIL: in vitro and in vivo evaluation. Mol Cancer Ther 2009; 5: 1387-97.

14 Wang G, Li G, Liu H, Yang C, Yang X, Jin J, et al. E1B 55-kDa deleted, Ad5/F35 fiber chimeric adenovirus, a potential oncolytic agent for B-lymphocytic malignancies. J Gene Med 2009; 11: 477-85.

15 Kruyt FA, Curiel DT. Toward a new generation of conditionally replicating adenoviruses: pairing tumor selectivity with maximal oncolysis. Hum Gene Ther 2002; 13: 485-95.

16 Post DE, Khuri FR, Simons JW, Van Meir EG. Replicative oncolytic adenoviruses in multimodal cancer regimens. Hum Gene Ther 2003; 14: 933-46.

17 Libertini S, lacuzzo I, Ferraro A, Vitale M, Bifulco M, Fusco A, et al. Lovastatin enhances the replication of the oncolytic adenovirus dI1520 and its antineoplastic activity against anaplastic thyroid carcinoma cells. Endocrinology 2007; 148: 5186-94.

18 Kantarjian HM, Talpaz M, Santini V, Murgo A, Cheson B, O'Brien SM. Homoharringtonine: history, current research, and future direction. 
Cancer 2001; 92: 1591-605.

19 Feldman E, Arlin Z, Ahmed T, Mittelman A, Puccio C, Chun H, et al. Homoharringtonine is safe and effective for patients with acute myelogenous leukemia. Leukemia 1992; 6: 1185-88.

20 Feldman EJ, Seiter KP, Ahmed T, Baskind P, Arlin ZA. Homoharringtonine in patients with myelodysplastic syndrome (MDS) and MDS evolving to acute myeloid leukemia. Leukemia 1996; 10: 40-2.

21 O'Brien S, Kantarjian H, Keating M, Beran M, Koller C, Robertson LE, et al. Homoharringtonine therapy induces responses in patients with chronic myelogenous leukemia in late chronic phase. Blood 1995; 86: 3322-6.

22 Warrell RP Jr, Coonley CJ, Gee TS. Homoharringtonine: An effective new drug for remission induction in refractory non-lymphoblastic leukemia. J Clin Oncol 1985; 3: 617-21.

23 Kang MH, Wan Z, Kang YH, Sposto R, Reynolds CP. Mechanism of synergy of $\mathrm{N}$-(4-hydroxyphenyl) retinamide and $\mathrm{ABT}-737$ in acute lymphoblastic leukemia cell lines: Mcl-1 inactivation. J Natl Cancer Inst 2008; 100: 580-95.

24 Chou TC, Motzer RJ, Tong Y, Bosl GJ. Computerized quantitation of synergism and antagonism of taxol, topotecan, and cisplatin against human teratocarcinoma cell growth: a rational approach to clinical protocol design. J Natl Cancer Inst 1994; 86: 1517-24.

25 Chu RL, Post DF, Khuri FR, Van Meir EG. Use of replicating oncolytic adenovirus in combination therapy for cancer. Clin Cancer Res 2004; 10: 5299-312.

26 Pan JJ, Zhang SW, Chen CB, Xiao SW, Sun Y, Liu CQ, et al. Effect of recombinant adenovirus-p53 combined with radiotherapy on longterm prognosis of advanced nasopharyngeal carcinoma. J Clin Oncol 2009; 27: 799-804.

27 Everts B, van der Poel HG. Replication-selective oncolytic viruses in the treatment of cancer. Cancer Gene Ther 2005; 12: 141-61.

28 Cody JJ, Douglas JT. Armed replicating adenoviruses for cancer virotherapy. Cancer Gene Ther 2009; 16: 473-88.

29 Kantarjian H, Keating M, McCredie K, Koller CA, McCredie KB,
Freireich EJ. Phase II study of homoharringtonine in refractory acute myelogenous leukemia. Cancer 1989; 63: 813-7.

30 Jin J, Jiang DZ, Mai WY, Meng HT, Qian WB, Tong HY, et al. Homoharringtonine in combination with cytarabine and aclarubicin resulted in high complete remission rate after the first induction therapy in patients with de novo acute myeloid leukemia. Leukemia 2006; 20 : 1361-7.

31 Luo CY, Tang JY, Wang YP. Homoharringtonine: a new treatment option for myeloid leukemia. Hematology 2004; 9: 259-70.

32 Karakas T, Maurer U, Weidmann E, Miething CC, Hoelzer D, Bergmann L. High expression of bcl-2 mRNA as a determinant of poor prognosis acute myeloid leukemia. Ann Onc 1998; 9: 159-65.

33 Addeo R, Caraglia M, Baldi A, D'Angelo V, Casale F, Crisci S, et al. Prognostic role of bcl-xL and p53 in childhood acute lymphoblastic leukemia (ALL). Cancer Biol Ther 2005; 4: 32-8.

34 Coustan-Smith E, Kitanaka A, Pui CH, McNinch L, Evans WE, Raimondi $\mathrm{SC}$, et al. Clinical relevance of BCL-2 overexpression in childhood acute lymphoblastic leukemia. Blood 1996; 87: 1140-6.

35 Guo BC, Xu YH. Bcl-2 over-expression and activation of protein kinase $C$ suppress the trail-induced apoptosis in Jurkat $T$ cells. Cell Res 2001; 11: 101-6.

36 Lamothe B, Aggarwal BB. Ectopic expression of $\mathrm{Bcl}-2$ and $\mathrm{Bcl}-\mathrm{xL}$ inhibits apoptosis induced by TNF-related apoptosis-inducing ligand (TRAIL) through suppression of caspases-8, 7, and 3 and BID cleavage in human acute myelogenous leukemia cell line $\mathrm{HL}-60$. J Interferon Cytokine Res 2002; 22: 269-79.

37 Ashkenazi A, Pai RC, Fong S, Leung S, Lawrence DA, Marsters SA, et al. Safety and antitumor activity of recombinant soluble Apo2 ligand. J Clin Invest 1999; 104: 155-62.

38 Jazirehi AR, Ng CP, Gan XH, Schiller G, Bonavida B. Adriamycin sensitizes the adriamycin-resistant 8226/Dox40 human multiple myeloma cells to Apo2L/tumor necrosis factor-related apoptosisinducing ligand-mediated (TRAIL) apoptosis. Clin Cancer Res 2001; 7: 3874-83. 